\title{
Acute Spinal Cord Injury Alters the Bioavailability of Oral and Intraperitoneal Cyclosporine-A in Contused Rats
}

\author{
A. Ibarra, R. Kretschmer, G. Guizar-Sahagun, H. Salgado-Ceballos, I. Grijalva, \\ F. Flores-Murrieta, G. Castañeda-Hernandez, A. Odor, R.M. Lopez, R. Franco-Bourland, \\ A.L. Espitia and I. Madrazo \\ Camina Research Center, IMSS, UNAM, CINVESTAV, INNSZ México, D.F., México
}

Pharmacokinetics (PK) of drugs such as gentamicin and theophylline is altered in humans with spinal cord (SC) injury (Segal, 1985). These observations led us to suspect that the PK of cyclosporine-A (CsA) might be altered in experimental SC injury. Our goal was to establish the best immunosuppressive strategy with CsA for our rat model of traumatic SC injury, in order to control the autoimmune response seen after trauma and to control the immune response to tissue grafts in transplanted $\mathrm{SC}$-lesioned rats. We determined the PK of CsA, administered in a single dose of $10 \mathrm{mg} / \mathrm{kg}$ b.w., in three groups of 10 rats each, as follows: Group A: acutely SC-lesioned rats. Five rats were given the drug orally and five received the drug intraperitoneally one day after injury. Group B: chronically SC-lesioned rats. Five rats were given CsA orally and five received the drug intraperitoneally 7-9 weeks after injury. Group C: sham-lesioned rats. Five rats were given CsA orally and five received the drug intraperitoneally one day after laminectomy. Blood samples were collected from the tail of each anesthetized rat before and 2, 4, 6, 12 and 24 hours after drug administration. The serum levels of CsA were determined by RIA, and the PK parameters assessed were: maximum serum concentration $\left(\mathrm{C}_{\max }\right)$, time to reach maximum serum concentration $\left(\mathrm{t}_{\max }\right)$ and area under the

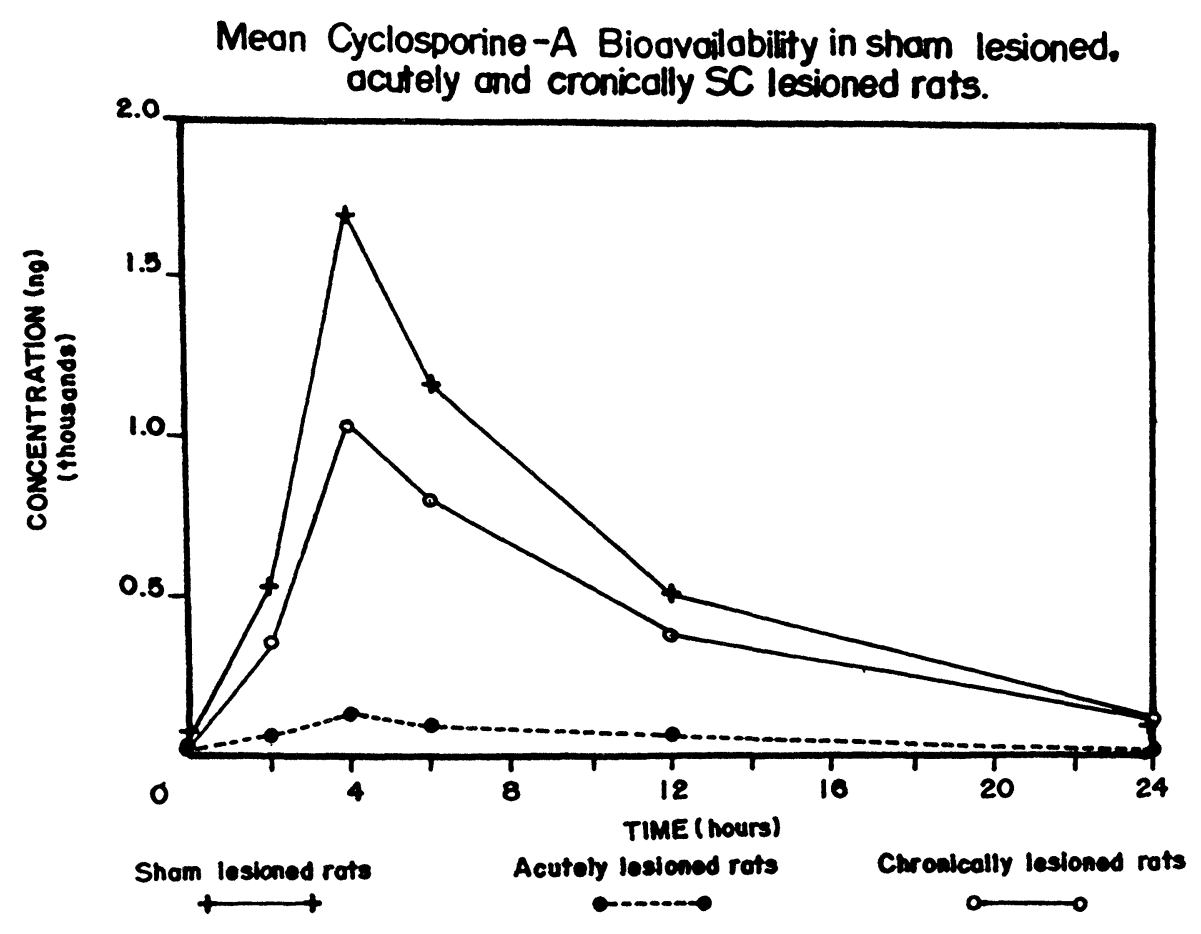


concentration-time curve (AUC). The results showed that CsA bioavailability was altered in acutely SC-lesioned rats. After oral administration, the bioavailability of CsA was statistically significantly reduced; serum levels were barely detectable after 12 hours. After intraperitoneal administration this group of lesioned rats showed an increase in CsA bioavailability compared with the sham-lesioned and the chronically SC-lesioned rats.

Altered bioavailability after oral administration of CsA could be attributed to the delayed gastric emptying secondary to spinal cord injury resulting in a deficient absorption of CsA in the upper small intestine. The altered bioavailability after intraperitoneal administration may be attributed to SC injury disturbances, such as vessel dilatation by dominant parasympathetic action, resulting in an increased drug absorption. On the other hand chronically SC-lesioned rats showed a CsA bioavailability profile similar to that observed in sham-lesioned animals, after both oral and intraperitoneal administration; the differences in the CsA bioavailability were not statistically significant. This may indicate that the recovery in the gastrointestinal and vessel motor functions tends towards normality in chronically SC-lesioned rats.

Finally, sham-lesioned rats showed similar bioavailability profiles to those of healthy rats, as reported by other authors (Wassef et al., 1985).

The differences observed in CsA bioavailability in SC-lesioned rats must be taken into consideration in order to establish an adequate immunosuppressive strategy. 

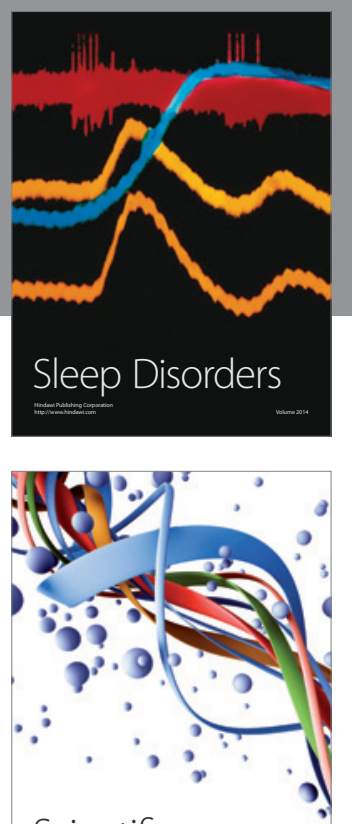

Scientifica
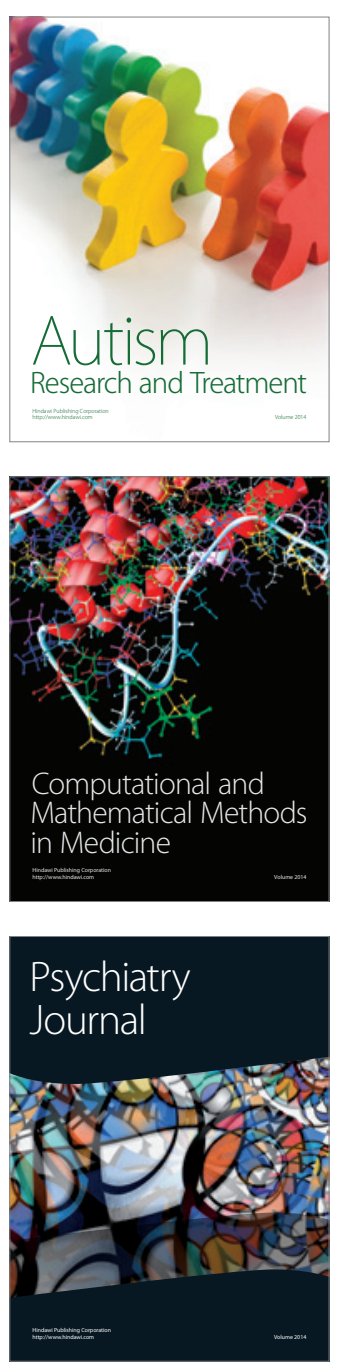
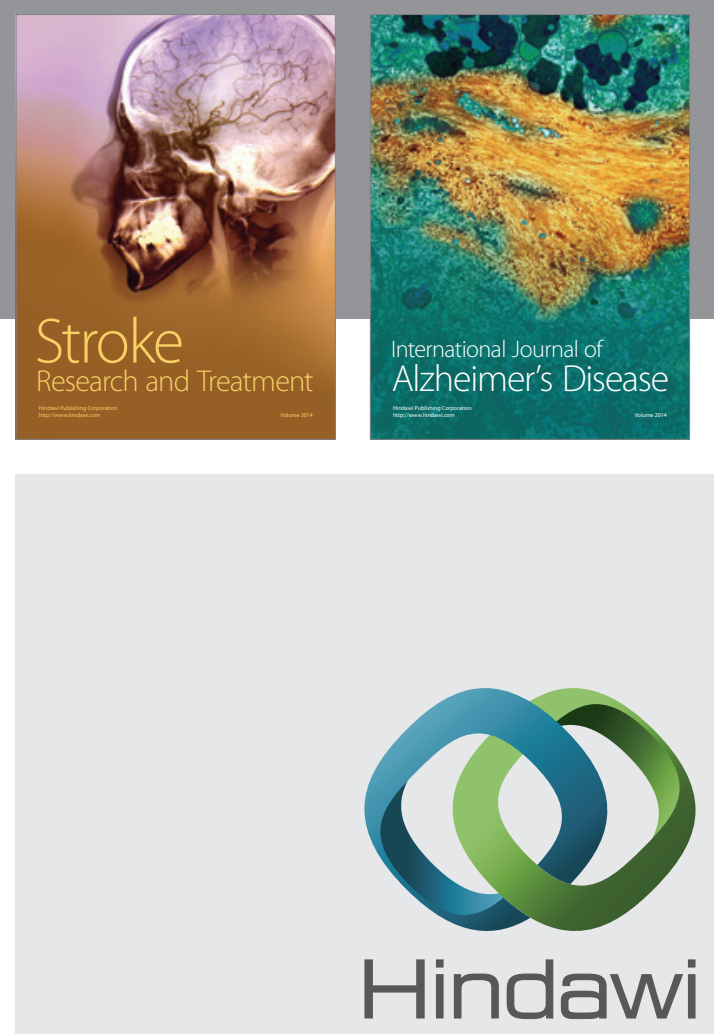

Submit your manuscripts at

http://www.hindawi.com
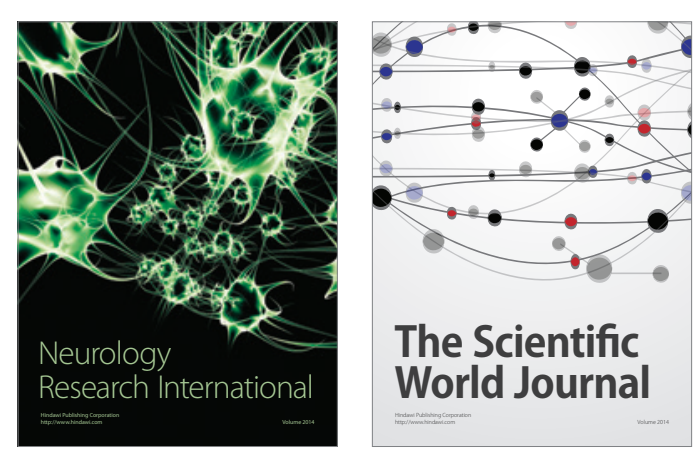

The Scientific World Journal

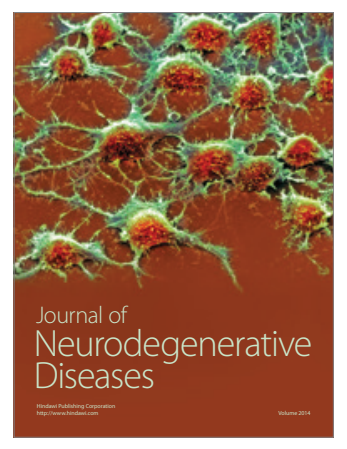

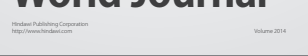

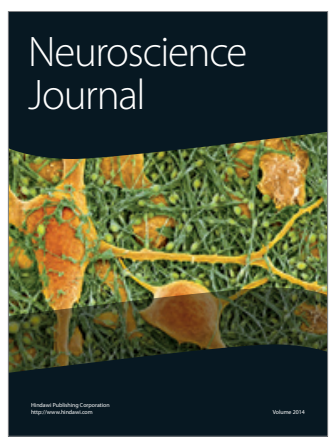

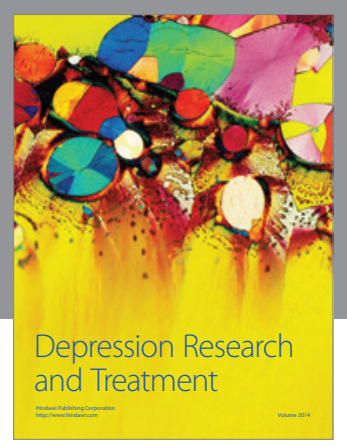
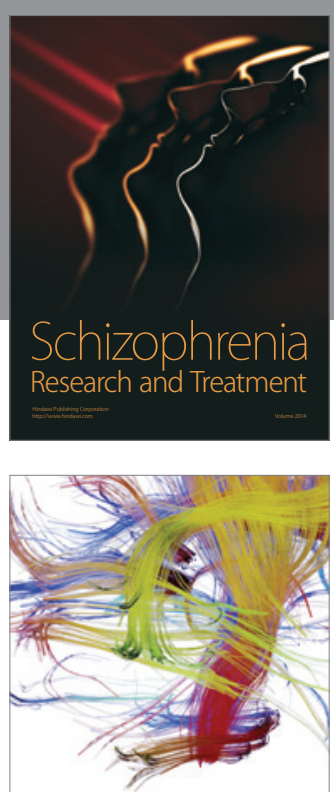

Brain Science

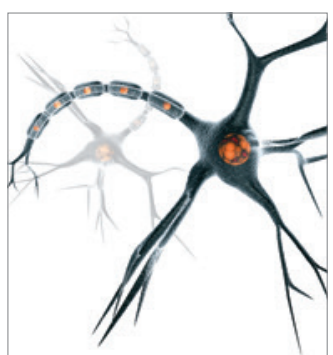

Neural Plasticity
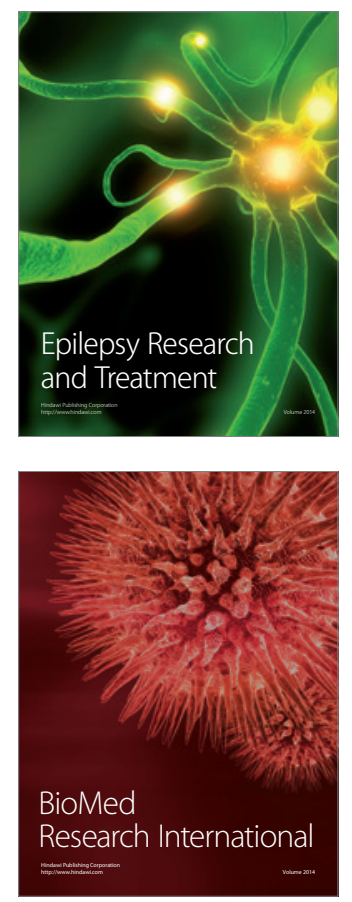

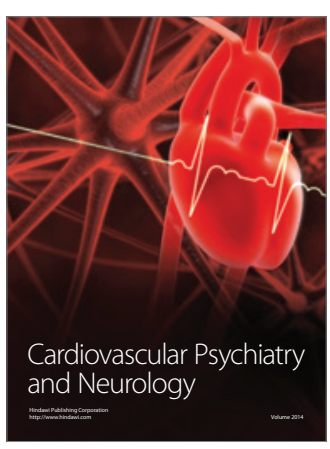

Parkinson's

Disease
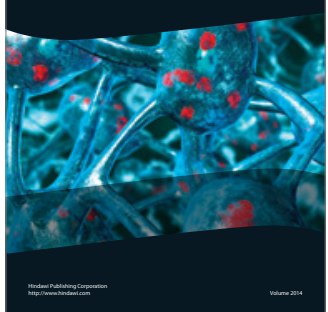